\title{
The Human Body Characteristics as a Signal Transmission Medium for Intrabody Communication
}

\author{
Namjun Cho, Student Member, IEEE, Jerald Yoo, Student Member, IEEE, Seong-Jun Song, Student Member, IEEE,
} Jeabin Lee, Student Member, IEEE, Seonghyun Jeon, Student Member, IEEE, and Hoi-Jun Yoo, Senior Member, IEEE

\begin{abstract}
The human body characteristics as a signal transmission medium are studied for the application to intrabody communication. The measurements of the body channel cover the frequency range from $100 \mathrm{kHz}$ to $150 \mathrm{MHz}$ and the distance on the body up to $1.2 \mathrm{~m}$. A distributed $R C$ model is developed to analyze the large variation of the channel properties according to the frequency and channel length. The simulation results using the channel model match well with the measurements in both the frequency and time domains. The effect of the ground plane to the body channel transceivers is also investigated and an empirical formula for the minimum ground size is obtained. Finally, the amount of the electromagnetic radiation due to the body antenna effect is measured. With regards to the Federal Communications Commission regulations, the proper frequency range for the intrabody communication is determined to satisfy given bit error rate requirements.
\end{abstract}

Index Terms-Body antenna effect, body channel model, distributed $R C$ model, human body communication, intrabody communication, near-field coupling.

\section{INTRODUCTION}

$\mathbf{I}$ NTRABODY communication is a new method to connect mobile devices on and inside the human body. This technique of using the human body as a signal transmission medium has many advantages over the conventional RF approaches. Since its operation is based on near-field coupling, most of the signal from the transmitter is confined to the body area without interference with external RF devices. Since the communication frequency can be lowered without enlarging antenna size, the power consumption of the transceiver is also much reduced compared to Bluetooth and Zigbee radios [1].

Many groups have studied intrabody communication systems, and the performance of such systems in data rate, operational distance, and size, has been improved. The initial prototypes of the transceiver used narrowband communication schemes, and their data rate was limited to several kilobits per second [2]-[4].

In addition, most of them had a bulky ground plane to enhance the coupling return path of the near-field. In contrast, the transceivers in newer systems use wide bandwidth to increase data rate, and avoid using a large ground plane by stabilizing the received signal with an electrooptic sensor or by enhancing

Manuscript received October 24, 2006; revised January 8, 2007.

The authors are with the Division of Electrical Engineering, Department of Electrical Engineering and Computer Science, Korea Advanced Institute of Science and Technology, Daejeon 305-701, Korea (e-mail: buzm@eeinfo.kaist.ac.kr; jerald@eeinfo.kaist.ac.kr; tornado@eeinfo.kaist.ac.kr; jeabin@eeinfo.kaist.ac.kr; shjeon@eeinfo.kaist.ac.kr;).

Digital Object Identifier 10.1109/TMTT.2007.895640

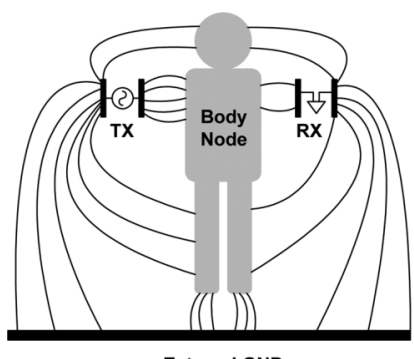

External GND

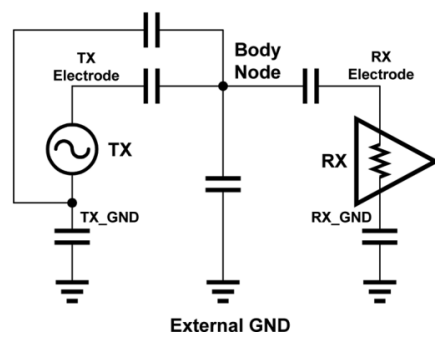

Fig. 1. Conventional circuit model of the body channel.

the receiver sensitivity [5], [6]. There are also other approaches to send a modulated current by using two electrodes at the transmitter and induce voltage differences around it with no consideration of the external ground [7].

Fig. 1 shows a conventional circuit model to describe the near-field coupling mechanism around the human body [1], [2]. The signal is transmitted between the body channel transceivers by making a current loop, which is composed of the transmitter electrode, the body channel, the receiver electrode, and the capacitive return path through the external ground. In this model, the human body is modeled as a perfect conductor, and the electric couplings among the electrodes of the transceiver, body, and environment are modeled as capacitors. The approximation of the human body as a single node is quite useful when the impedance of the return path is very large compared to that of the body channel [1]. However, as the communication frequency increases for a high data rate, the coupling capacitances of the return path have less effect and the body impedance cannot be ignored. As the transmission length of the body channel increases, both the resistance of the body and the coupling capacitance to the external ground increase. These elements cause signal loss at the receiver, and its amount depends on the channel length. In this paper, a distributed $R C$ model of the human body is developed to analyze the signal transmission through the human body at higher frequencies. It consists of the cascaded unit blocks with an $R C$ parallel network and shunt capacitors, which model the electrical couplings across the human body. By selecting the parameters of the composing passive elements to form a T-shaped human subject with cascaded unit blocks, the electrical circuit model of the body channel can be easily obtained. The simulated results based on the proposed model match well with the measurement results of $S_{21}$-parameters and the transient responses of the test body channel. In addition, the body channel transceivers with different sizes of the ground planes are measured to investigate their effects on the received power level. 
Although data transmission with a high-frequency carrier can exploit the strong return path coupling and high data rate, it has an interference problem. Even though the communication range by the near-field coupling is limited to the near body area, the high-frequency carrier may radiate electromagnetic waves into the air. The radiation effect limits the body channel transceivers in terms of frequency and transmission power. To evaluate these limits, the strength of the $E$-field is measured around a human body with a body channel transmitter attached on the skin. The maximum power level and the optimum frequency range for the body channel communication are determined, with consideration for Federal Communications Commission (FCC) field strength regulations.

\section{BODY CHANNEL CHARACTERISTICS}

\section{A. Distributed RC Model}

Fig. 2(a) shows a near-field coupling model of the human body, which consists of three cylinders. The larger one among them models the human torso of which height and diameter are 140 and $30 \mathrm{~cm}$, respectively. Two slender cylinders approximate two arms with $10-\mathrm{cm}$ diameter and $60-\mathrm{cm}$ length. The three cylindrical models are virtually segmented into unit blocks, which are 10-cm long. For each unit block, its complex impedance is calculated from the Gabriel's experimental results [8] and the coupling capacitance to the external ground is calculated based on the Zimmerman's method [2]. Although the electrical properties of the human body are highly dependent on the frequency and the locations of the body, the conductivity $(\sigma)$ is within $0.1-1 \mathrm{~S} / \mathrm{m}$ and the relative permittivity $\left(\varepsilon_{r}\right)$ varies from 50 to 200 in the frequency range of $1-150 \mathrm{MHz}$, which we are interested in. Under $1 \mathrm{MHz}$, the electrical properties have little effect on the body channel characteristics since the strength of the return path is extremely weak. Therefore, the electrical model of the unit block can be a discrete $R C$-parallel network with constant parameters. Assuming the unit block is isotropic and its cross sections are equipotential, the resistance and capacitance can be obtained from the simple equations $-R=L / \sigma A$ and $C=\varepsilon_{r} \varepsilon_{0} A / L$, where $L$ and $A$ are the length and the cross-sectional area of a unit block, respectively.

The coupling capacitance $\left(C_{C}\right)$ to the external ground is computed by approximating the unit block as the part of a conductive sphere in the free space [2]. The unit capacitance value is obtained with the assumption that a person stands in an open space. If any large conductive object that can provide a coupling path with the human is present nearby, the capacitance value will further increase. The air coupling capacitances between the transceiver grounds and the unit block are highly affected by the body configurations. In most cases, their values are very small, ranging from 10 to $100 \mathrm{fF}$ with little effect on the channel property. By cascading the multiple $R C$ blocks to construct the three cylinder models and combining them, a complete circuit model of the human body can be obtained, as shown in Fig. 2(b). The $R$ and $C$ values in the distributed network are calculated from the electrical properties in $10-60 \mathrm{MHz}$ and listed together. To consider the channel responses at the various locations on the body, the receiver model can be placed at the corresponding nodes of the circuit model. Since each sub $R C$ circuit is for the 10-cm unit

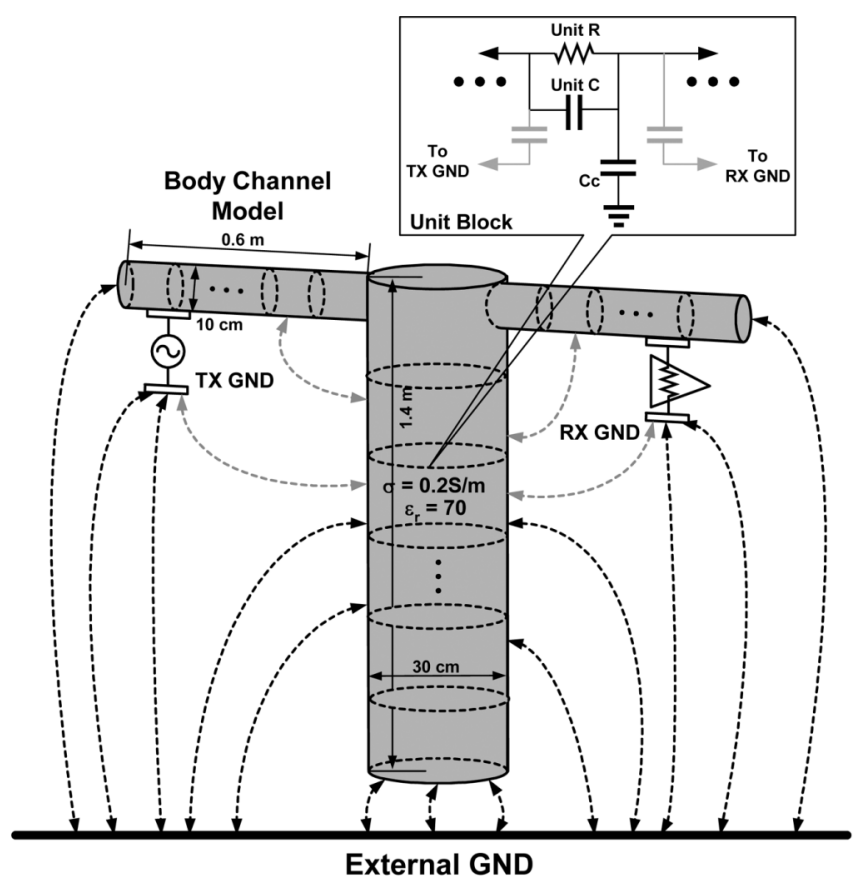

(a)

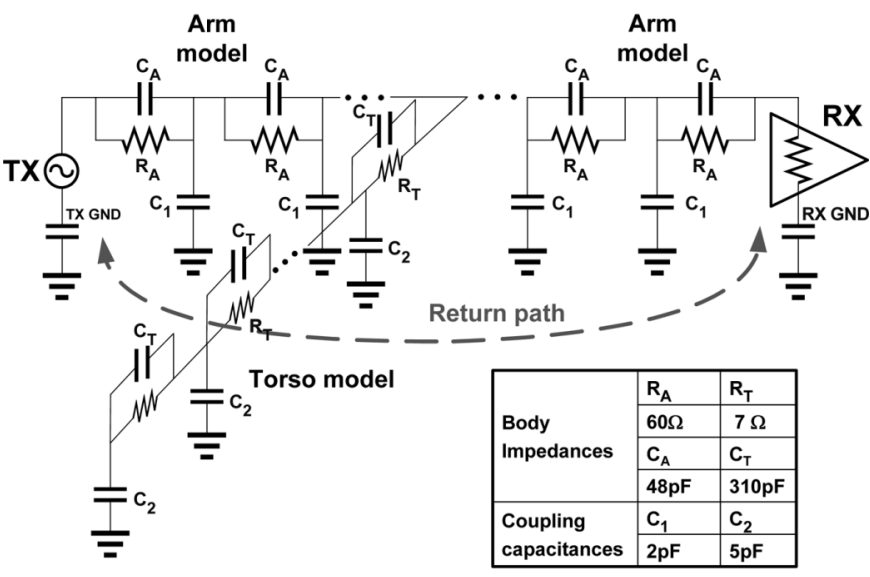

(b)

Fig. 2. Proposed circuit model of the body channel. (a) Near-field coupling model of the human body. (b) Complete circuit model of the body channel.

block, $N$-stage cascaded blocks are equivalent to the $N \times 10 \mathrm{~cm}$ channel length. In the distributed network, the major return path is formed by the electrical coupling between the transmitter and receiver grounds through the external ground. Therefore, using a large ground plane or a special electrode for the return path loop is advantageous to enhance the SNR of the received signal. However, since a large ground plane is difficult to implement on the body channel transceiver, it is useful to find a lower limit on the plane size.

\section{B. Measurement Setup}

A careful setup is required to measure the body channel characteristics because the ground plane must be considered with special attention. Since the return path across the body channel transceivers is formed by the electrical couplings, the earthgrounded instruments, such as the signal generator and the network analyzer at the transmitter part, do not reproduce a real 


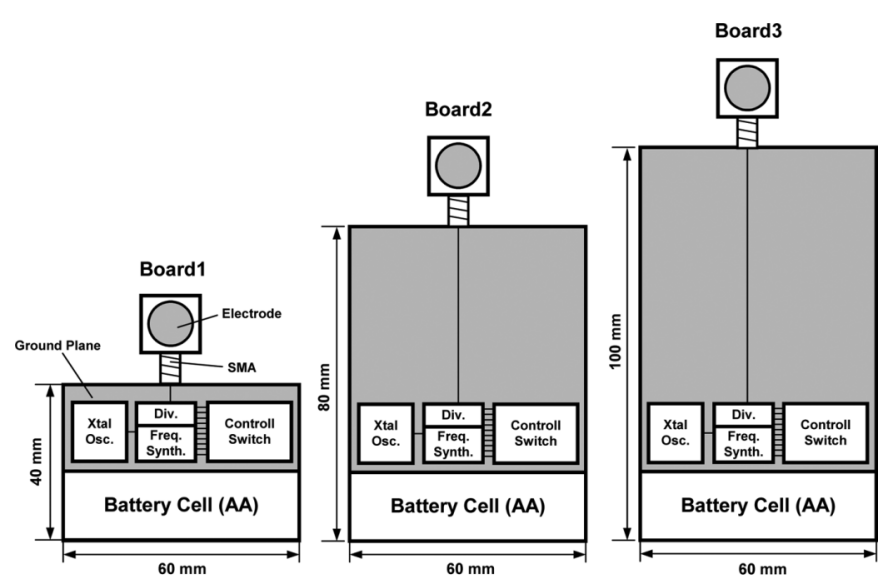

(a)

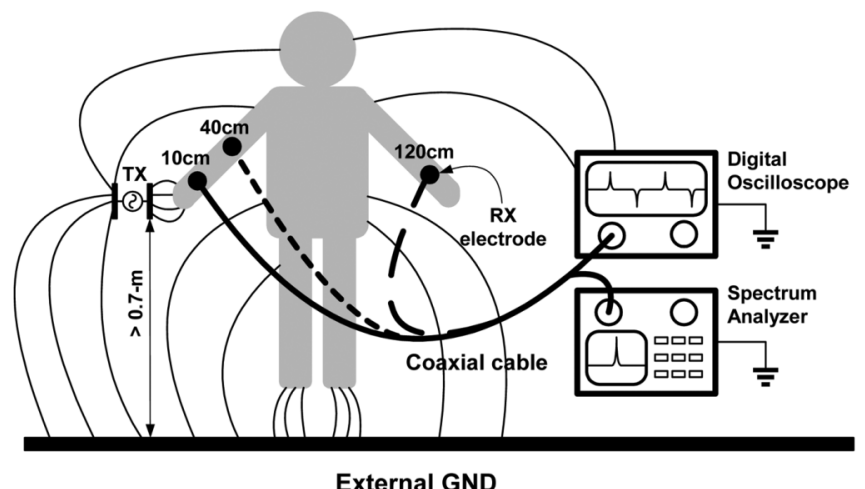

(b)

Fig. 3. Experimental setup for the body channel measurement. (a) Transmitter boards. (b) Experimental configuration.

situation. To simulate an actual near-field coupled environment, a battery-powered signal generator is used with a programmable frequency synthesizer. Fig. 3(a) shows the structure of the designed transmitter board. The frequency synthesizer and divider are combined to provide frequencies from $100 \mathrm{kHz}$ to $150 \mathrm{MHz}$ with $50 \%$ duty cycle, and the available output power from the transmitter is fixed to $3 \mathrm{dBm}$. A circular electrode with $1.5-\mathrm{cm}$ diameter is used to interface electric power to the skin. This electrode is also used at the receiving part. The material for the electrode has little effect on the magnitude of the received signal if it is a conductor [9]. The entire plane of the transmitter board is treated as the ground. To investigate the ground effect, the area of the ground plane is varied from 24 to $60 \mathrm{~cm}^{2}$. The minimum height of the plane is determined by the AA-sized battery. Fig. 3(b) is the experimental configuration for the body channel measurement. The height of the human subject is $1.8 \mathrm{~m}$. During the measurement, various poses of the subject-standing with two arms outstretched, attached at his side, sitting down, etc. - are considered and multiple data are collected for the same frequency and distance. The transmitting electrode is attached to the right hand of the human body. The transmitter is separated from the external ground by more than $0.7 \mathrm{~m}$. At the receiver side, a spectrum analyzer or an oscilloscope is connected at the receiving electrode through a coaxial cable to measure the signal. The distances between the transmitter and receiver are set to 10,40 , and $120 \mathrm{~cm}$ by changing the receiver locations

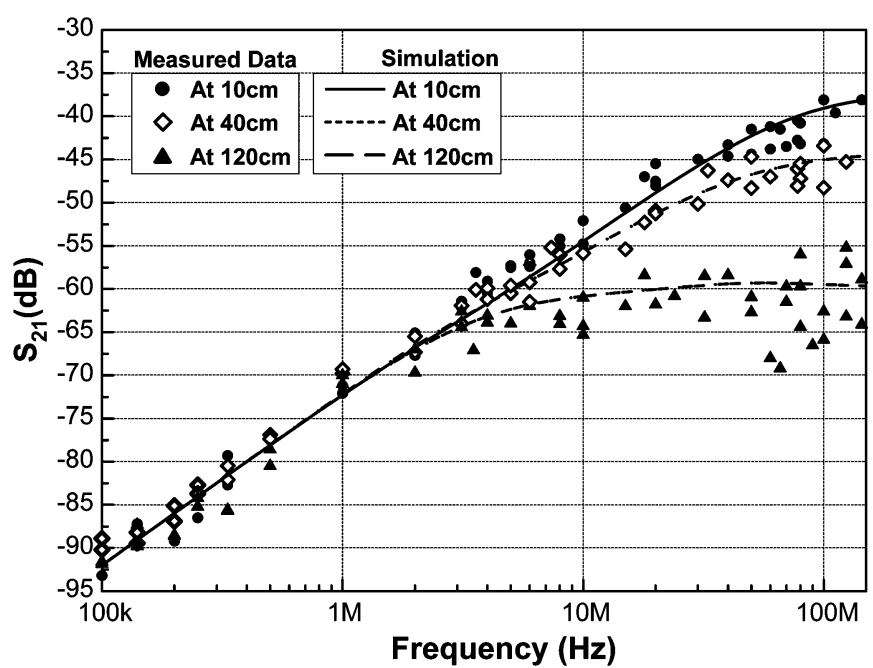

(a)

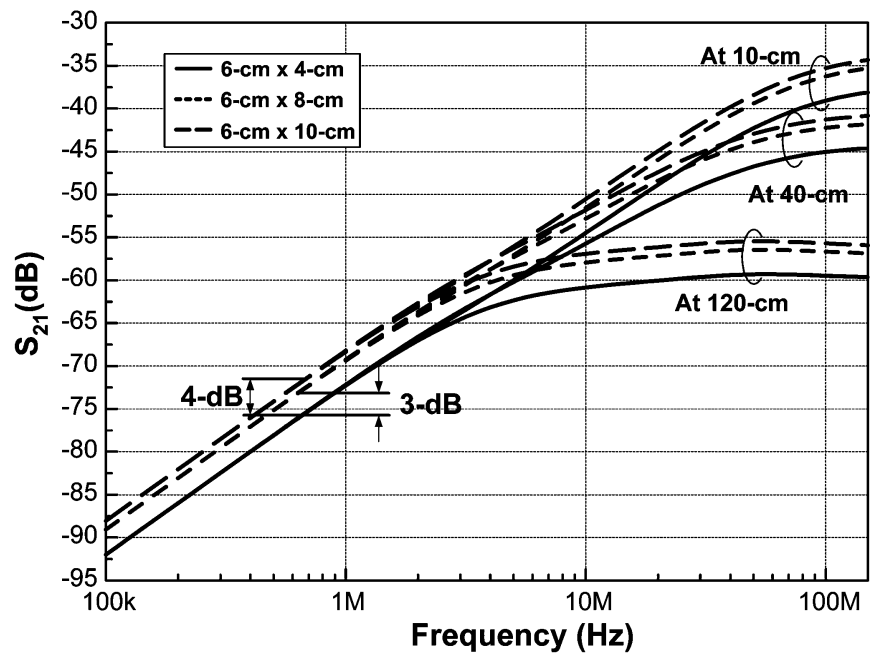

(b)

Fig. 4. Measured $S_{21}$-parameters of the body channel. (a) With $6 \mathrm{~cm} \times 4 \mathrm{~cm}$ ground size. (b) With different ground sizes.

from the right arm holding the transmitter to the left arm across the chest. With these settings, the receiver shares its ground with the earth-grounded instruments so that the strength of the return path is rather increased. The increment is less than $6 \mathrm{~dB}$ when the transmitter and receiver have the same size. This increment is considered in the following analysis to determine the minimum ground size of the transceiver.

\section{Measurement Results}

The channel characteristics of the human body are investigated in both the frequency and time domains. For the frequency-domain analysis, $S_{21}$-parameters of the body channel are measured. The transient step response through the body is also measured for the time-domain channel characteristics.

The graphs in Fig. 4 show the measured $S_{21}$-parameters with respect to the transmitted signal frequency. The effects of the channel distance and the transmitter ground size are considered, and the simulated $S_{21}$-parameters with the distributed $R C$ model are also included. The graph shows that below $4 \mathrm{MHz}$, the body channel is relatively deterministic, with at most $5 \mathrm{~dB}$ 

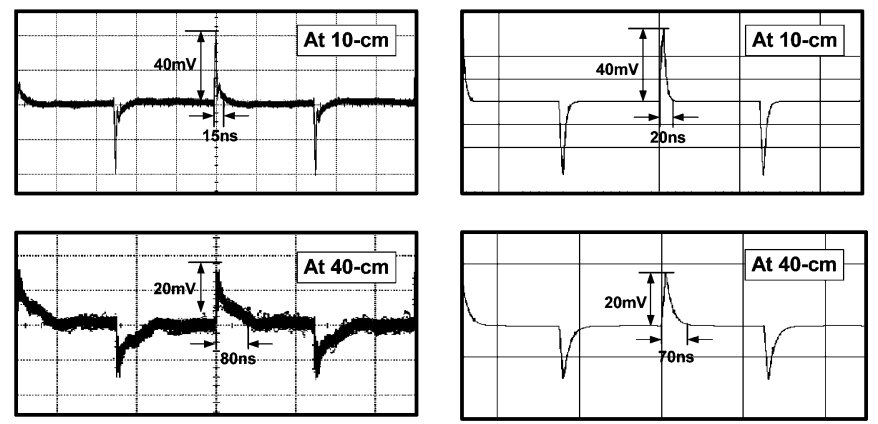

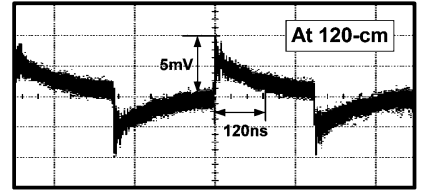

(a)

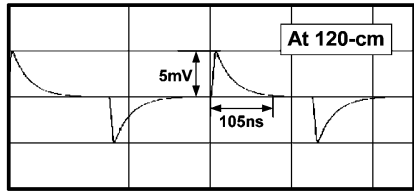

(b)
Fig. 5. Step response of the body channel. (a) Measured step response. (b) Simulated step response.

of deviation regardless of the distance. This is because the body impedance is negligible compared to the impedance of the return path, and a very small amount of power is lost from the body to the external ground. In this region, the body channel looks like a high-pass filter due to the capacitive return path [6]. This property implies that better SNR and power savings are possible by using a high frequency for the body channel communication. However, beyond $10 \mathrm{MHz}$, the channel distance has a great effect on the transferred power. When the distance between the transceivers is short enough, the channel remains as the high-pass filter since the power loss on the signal path is still low. As the channel length increases, the capacitive coupling between the body and the external ground becomes larger, and induces larger signal loss. Especially at $120-\mathrm{cm}$ distance, much of the transmitted power is lost to the external ground through the torso. This effect becomes more evident at high frequencies. As a result, the $S_{21}$ curve of the body channel is bent gradually downward and its cutoff frequency is lowered with the distance. The large fluctuation of the $S_{21}$ values at the 120 -cm distance comes from the different poses of the human body. Since the air coupling between the transmitter and receiver increases with frequency, the signal radiated into the air may be scattered on the surface of the skin and may arrive at the receiver with shifted phases. The scattered signal can be superimposed on the signal through the body constructively or destructively, and it causes large variations of the received power at high frequencies. The graph presented in Fig. 4(b) shows that the size of the ground plane is in proportion with the $S_{21}$ values. When the ground area increases by 2 and 2.5 times, the $S_{21}$-parameters grow by 3 and $4 \mathrm{~dB}$, respectively. The graph also shows that the ground size of the transceiver has little effect on the overall shape of the channel response.

Fig. 5 shows the transient channel response of a $2-\mathrm{MHz}$ square wave with $3.5-\mathrm{V}$ amplitude and 10-ns transition time. The output waveform has positive and negative pulses, and at $10-\mathrm{cm}$ distance, it has $15-\mathrm{ns}$ duration and $40-\mathrm{mV}$ peak. As the distance on the body increases, the peak value of the pulse is lowered and its width becomes wider. These results correspond well to the results of the frequency-domain analysis, i.e., the high-frequency components of the signal attenuate drastically with the distance.

In summary, the body channel characteristics highly depend on the distance, ground size, and frequency of the signal. If the transmitting power $\left(P_{\mathrm{TX}}\right)$, receiver sensitivity $\left(S_{\mathrm{RX}}\right)$, carrier frequency $(f)$, and the channel length $(D)$ are given, the minimum ground size $\left(A_{\min }\right)$ can be obtained as

$$
\begin{gathered}
A_{\text {min }}=\frac{S_{\mathrm{RX}}}{P_{\mathrm{TX}}} \frac{\left(1+f / f_{0}\right)^{P}}{\left(1+f / 2 f_{0}\right)^{2}(K f)^{2}}, \quad K=3 \times 10^{-9} ; \\
f_{0}=\frac{10 \times 10^{6}}{D} ; \quad P= \begin{cases}4, & D>0.8 \\
3, & \text { else. }\end{cases}
\end{gathered}
$$

For example, if a body channel transmitter is to send $1-\mathrm{Mb} / \mathrm{s}$ frequency shift-keying (FSK) modulated data with $-5 \mathrm{dBm}$ of power using a carrier frequency from 10 to $150 \mathrm{MHz}$, and if a receiver's sensitivity is $-70 \mathrm{dBm}$ for the bit error rate (BER) of $10^{-6}$ [10], then a ground plane of at least $32-\mathrm{cm}^{2}$ size is sufficient regardless of the location of the transceivers on the body.

\section{BODY ANTENNA EFFECT}

Using high frequencies for the body channel communication is obviously beneficial to obtain a high SNR and data rate. However, the rapid change of the electric field induces electromagnetic radiation outside of the human body. At the high frequencies, the human body operates as an antenna radiating the signal power into the air. To investigate the proper frequency range for the body channel communication with little interference to the nearby RF devices, the $E$-field strength is measured around a human body with a body channel transmitter on the skin. The measured frequency range is from 1 to $150 \mathrm{MHz}$. The signal power is maintained at $3 \mathrm{dBm}$ for the entire frequency range. The size of the transmitter board is $6 \mathrm{~cm} \times 4 \mathrm{~cm}$, and the distance from the body to the antenna is $3 \mathrm{~m}$. At low frequencies under $30 \mathrm{MHz}$, a rod antenna of $1-\mathrm{m}$ height is utilized for measurements. At higher frequencies, a biconic dipole antenna, which has $0.5-\mathrm{m}$ diameter and $1.3-\mathrm{m}$ height, is used. Both of the receiving antennas are oriented vertically at $1 \mathrm{~m}$ above the ground plane. The signal received from the antenna is transferred to an electromagnetic interference (EMI) receiver to analyze the $E$-field strength. Measurements are performed in an anechoic chamber, which conforms to the emission measurement standards. The external dimensions of the chamber are $9.7 \mathrm{~m} \times 6.38 \mathrm{~m} \times 6 \mathrm{~m}$ and each internal wall is constructed with ferrite and hybrid absorbers. Fig. 6 shows the measured $E$-field pattern around the human body. The view direction of the person wearing the body channel transmitter on is set to $0^{\circ}$. As expected, the $E$-field magnitude increases with the transmitting frequency. The maximum radiated power is 30 times larger than that of the minimum power at $4 \mathrm{MHz}$. The radiation pattern of the transmitted signal is irregular and its directivity at the different frequencies is unpredictable. This is a common phenomenon in the near-field situation. In addition, there are many factors that affect the measurements-the complex shape of a human body, height differences of human beings, and various 


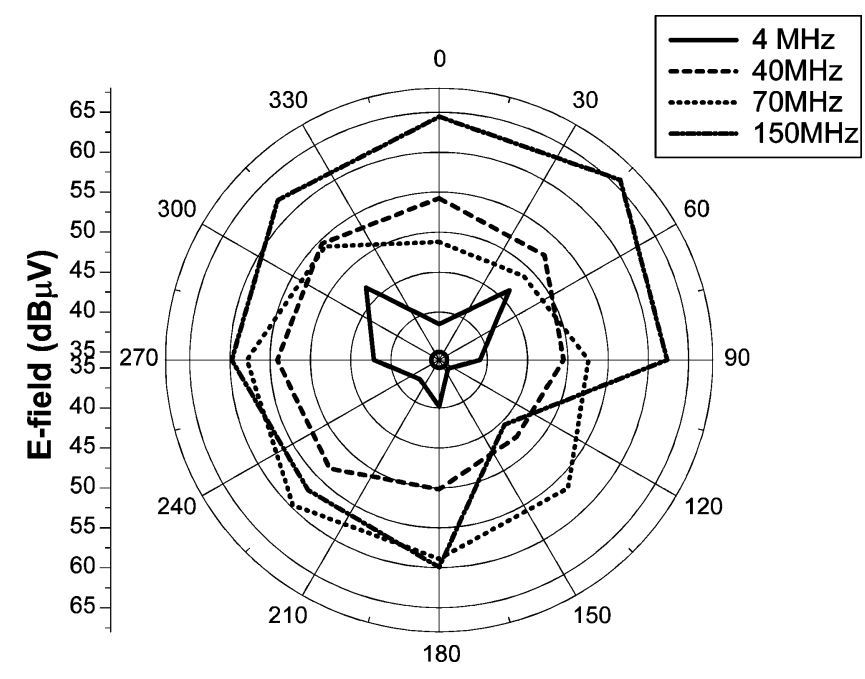

Fig. 6. $E$-field pattern around a person with a body channel transmitter.

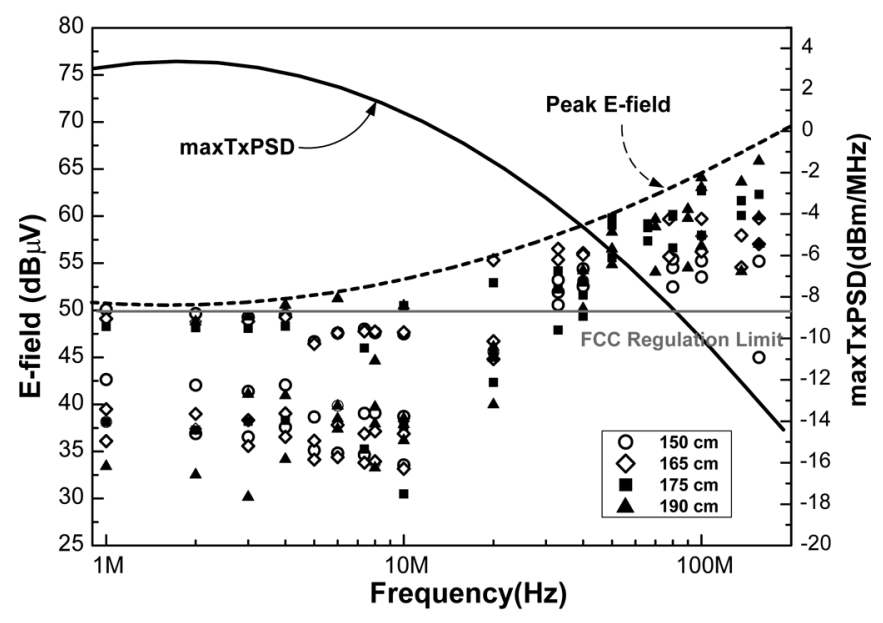

Fig. 7. Measured $E$-field data from multiple individuals.

poses of the person. Therefore, the $E$-field data from multiple individuals are measured and displayed in Fig. 7 for a statistical analysis. The people who participate in the experiment have different heights, ranging from 1.5 to $1.9 \mathrm{~m}$, and they are requested to take various poses. According to the measurement results, the $E$-fields are mainly affected by the poses of the experimental person and the directions of the receiving antenna at low frequencies. The heights of the human subjects make little difference. As the frequency increases, however, a taller person radiates larger electromagnetic power around him. This is because taller people have lower resonance frequencies, which are closer to the interested frequency region. From the measured data, we can determine the maximum power spectral density of the transmitted signal that does not violate the FCC regulations [11] at each frequency. As the frequency decreases, the transmitting power can be increased without violation of FCC regulations. However, the power attenuation through the body channel also increases in the low-frequency region, as shown in Fig. 4. These opposite trends imply that there is an optimal frequency range for the body channel communication to maximize the signal level at the receiver. To determine the optimal frequency band,

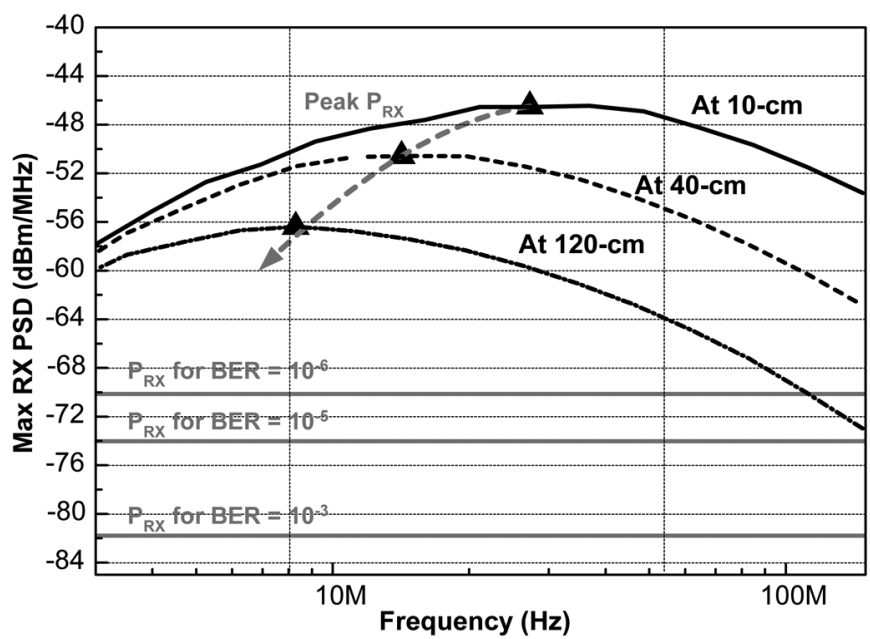

Fig. 8. Maximum RX power in the body channel communication.

the maximum power allowed at each frequency is applied to the body channel and the received power level is obtained, as shown in Fig. 8. At the 10-cm distance, the peak power spectral density is $-46 \mathrm{dBm} / \mathrm{MHz}$ at $30 \mathrm{MHz}$ and the upper $3-\mathrm{dB}$ frequency is $80 \mathrm{MHz}$. When the channel length increases, the bandwidth and signal power are diminished, limiting the communication channel capacity of the body. To evaluate the practical limits, BERs reported for low-power wireless receivers are also denoted in Fig. 8 [10], [12]. The BER data measured in the air channel can be applied to the body channel estimation because most of the noise signals such as electrocardiogram (ECG), electromyogram (EMG) and electroencephalogram (EEG) generated by a human body exist within $10 \mathrm{kHz}$ [13], and little noise consideration, except additive white Gaussian noise (AWGN) is required in the measured frequency region. According to the required BER, the available frequency band for the intrabody communication can be determined. For instance, voice transmission through the body channel would be successful under a BER of $10^{-3}$. In this case, any frequency range of the measurement can be utilized. If data packets of vital signals and private messages need to be transmitted through the body channel, the frequencies below $150 \mathrm{MHz}$ are recommended for a BER of $10^{-5}[14]$.

\section{CONCLUSION}

The body channel characteristics for intrabody communication are investigated. The $S_{21}$ values through the body channel are measured up to $150 \mathrm{MHz}$ and $1.2-\mathrm{m}$ distance. From the measurement results, the body channel shows high-pass filter characteristics under 4-MHz frequency due to the capacitive return path. Over $10 \mathrm{MHz}$, the body channel has a wide variation with the frequency and distance. A distributed $R C$ model is developed and verified by comparing the measurement data with simulation results in both the frequency and time domains. In addition, the effect of the ground size of the transceivers is investigated and an empirical formula for the minimum ground size is obtained. Finally, the electromagnetic radiation from a human body with a body channel transceiver on the skin is examined. From the $E$-field measurements, the limit on the output 


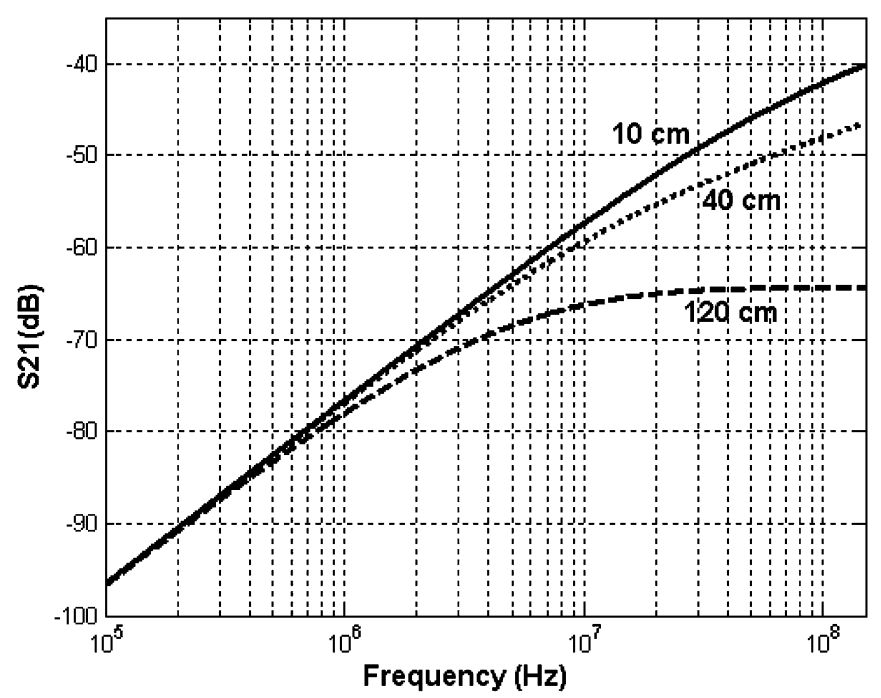

Fig. 9. Body channel characteristics from the empirical formula.

power of the transmitter is investigated with regard to FCC regulations. Through the maximum received power analysis, the body channel can guarantee the BER of $10^{-5}$ below $150 \mathrm{MHz}$, and better bit error performance can be achieved by lowering the communication frequency and shortening the channel distance.

\section{APPENDIX}

From the measurement results in Fig. 4(a), we can derive an empirical equation describing the body channel characteristics $(T)$ as

$$
\begin{gathered}
T=\frac{P_{\mathrm{RX}}}{P_{\mathrm{TX}}}=\frac{(K f)^{2}\left(1+f / 2 f_{0}\right)^{2}}{\left(1+f / f_{0}\right)^{P}} A_{G}, \quad K=3 \times 10^{-9} ; \\
f_{0}=\frac{10 \times 10^{6}}{D} ; \quad P= \begin{cases}4, & D>0.8 \\
3, & \text { else. }\end{cases}
\end{gathered}
$$

This equation includes the parameters that cause the channel variation-frequency $(f)$, channel distance $(D)$, and ground area $\left(A_{G}\right)$ of the transceiver. $K$ is the empirical coefficient and the value of $P$ depends on whether the receiver is located across the human chest or not. Fig. 9 graphically shows the channel responses of transceiver systems with $0.0024 \mathrm{~m}^{2}(6 \mathrm{~cm} \times 4 \mathrm{~cm})$ ground area $\left(A_{G}\right)$. Based on the empirical formula, we can decide the minimum ground size $\left(A_{\min }\right)$ of a body channel transceiver assuming that the transmitter's output power $\left(P_{\mathrm{TX}}\right)$ and the receiver's sensitivity $\left(S_{\mathrm{RX}}\right)$ are known as follows:

$$
A_{\min }=\frac{S_{\mathrm{RX}}}{P_{\mathrm{TX}}} \frac{\left(1+f / f_{0}\right)^{P}}{\left(1+f / 2 f_{0}\right)^{2}(K f)^{2}} .
$$

To get a numerical value from this equation, we refer the reader to the sensitivity data of a previously reported wireless receiver [10].

\section{REFERENCES}

[1] T. G. Zimmerman, "Personal area networks: Near-field intra body communication,” IBM Syst. J., vol. 35, no. 3-4, pp. 609-617, 1996.
[2] _ _ "Personal area networks (PAN): Near-field intra body communication," M.S. thesis, Media Lab., Massachusetts Inst. Technol., Cambridge, MA, 1995.

[3] K. Partridge, B. Dahlquist, A. Veiseh, A. Cain, A. Foreman, J. Goldberg, and G. Borriello, "Empirical measurements of intrabody communication performance under varied physical configurations," in User Interface Softw. Technol. Symp., Nov. 2001, pp. 183-190.

[4] N. Matsushita, S. Tajima, Y. Ayatsuka, and J. Rekimoto, "Wearable key: Device for personalizing nearby environment," in Int. Wearable Comput. Symp., Oct. 2000, pp. 119-126.

[5] M. Shinagawa, M. Fukumoto, K. Ochiai, and H. Kyuragi, "A nearfield-sensing transceiver for intrabody communication based on the electrooptic effect," IEEE Trans. Instrum. Meas., vol. 53, no. 6, pp. 1533-1538, Dec. 2004

[6] S.-J. Song, N. Cho, S. Kim, J. Yoo, and H.-J. Yoo, "A 2 Mb/s wideband pulse transceiver with direct-coupled interface for human body communications," in IEEE Int. Solid-State Circuits Conf. Tech. Dig., Feb. 2006, pp. 558-559.

[7] M. S. Wegmueller, M. O. Norbert Felber, N. Kuster, and W. Fichtner, "Galvanical coupling for data transmission through the human body," in Instrum. Meas. Technol. Conf., Apr. 2006, pp. 1686-1689.

[8] S. Gabriel, R. W. Lau, and C. Gabriel, "The dielectric properties of biological tissues: II. Measurements in the frequency range $10 \mathrm{~Hz}$ to 20 GHz," Phys. Med. Biol., pp. 2251-2269, Nov. 1996.

[9] K. Hachisuka, A. Nakata, T. Takeda, Y. Terauchi, K. Shiba, K. Sasaki, H. Hosaka, and K. Itao, "Development and performance analysis of an intra-body communication device," in Int. Solid-State Sens., Actuators, Microsyst. Conf., Jun. 2003, pp. 1722-1725.

[10] H. Darabi, J. Chiu, S. Khorram, H. J. Kim, Z. Zhou, H. Ming, B. Ibrahim, E. Geronaga, L. H. Tran, and A. Rofougaran, "A dual-mode 802.11b/Bluetooth radio in 0.35- $\mu \mathrm{m}$ CMOS," IEEE J. Solid-State Circuits, vol. 40, no. 3, pp. 698-706, Mar. 2005.

[11] FCC, Washington, DC, "Title 47 of the code of federal regulations: Part 15-Radio frequency devices," 2001.

[12] H. Komurasaki, T. Sano, T. Heima, K. Yamamoto, H. Wakada, I. Yasui, M. Ono, T. Miwa, H. Sato, T. Miki, and N. Kato, "A 1.8-V operation RF CMOS transceiver for 2.4-GHz-band GFSK applications," IEEE J. Solid-State Circuits, vol. 38, no. 5, pp. 817-825, May 2003.

[13] S. Arnon, D. Bhastekar, D. Kedar, and A. Tauber, "A comparative study of wireless communication network configurations for medical applications," IEEE Wireless Commun., vol. 10, no. 1, pp. 56-61, Feb. 2003.

[14] C. Chien, Radio System Design, Digital Radio Systems on a Chip, 1st ed. Norwell, MA: Kluwer, 2001.

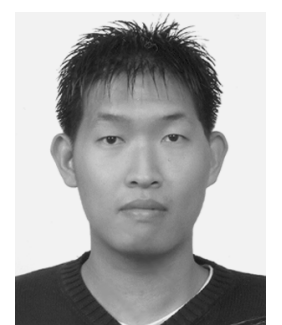

Namjun Cho (S'04) received the B.S. (summa cum laude) and M.S. degrees from the Korea Advanced Institute of Science and Technology (KAIST), Daejeon, Korea, in 2004 and 2006, respectively, and is currently working toward the Ph.D. degree at KAIST.

$\mathrm{He}$ has been involved with the development of UHF RF identification (RFID) tag chips integrated with environmental monitoring sensors and the low-power digital-to-analog converter for hearing-aid systems. His current research interests include low-power biomedical microsystems and wireless transceivers for body area networks.

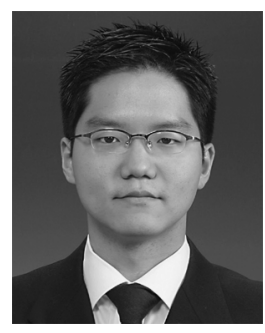

Jerald Yoo (S'05) received the B.S. and M.S. degrees in electrical engineering and computer science from the Korea Advanced Institute of Science and Technology (KAIST), Daejeon, Korea, in 2002 and 2007 , respectively, and is currently working toward the $\mathrm{Ph} . \mathrm{D}$. degree in electrical engineering and computer science at KAIST.

$\mathrm{He}$ has been involved with the development of the embedded processor for PRAM chips. His current research interests include energy-efficient body channel networks, low-power biomedical microsystems, and network processors for body area and sensor networks. 


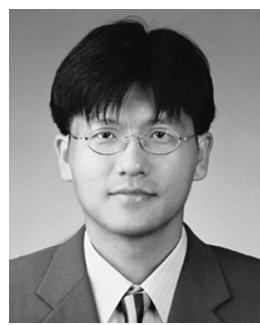

Seong-Jun Song (S'01) received the B.S. (summa cum laude) and M.S. degrees in electrical engineering and computer science from the Korea Advanced Institute of Science and Technology (KAIST), Daejeon, Korea, in 2001 and 2004, respectively, and is currently working toward the $\mathrm{Ph} . \mathrm{D}$. degree in electrical engineering and computer science at KAIST.

Since 2001, he has been a Research Assistant with KAIST, where he has been involved with the development of high-speed optical interface integrated circuits using submicrometer CMOS technology, phaselocked loops and clock and data recovery circuits for high-speed data communications, and RF CMOS integrated circuits for wireless communications. His current research interests include ultra low-power wearable/implantable biomedical microsystems and energy-efficient communication systems for body area and sensor networks.

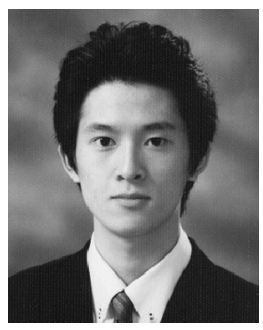

Jeabin Lee (S'07) received the B.S. degree (summa cum laude) in electrical engineering and computer science from the Korea Advanced Institute of Science and Technology (KAIST), Daejeon, Korea, in 2006, and is currently working toward the M.S. degree in electrical engineering and computer science at KAIST.

His research interest includes body channel communication systems and low-power circuit design.

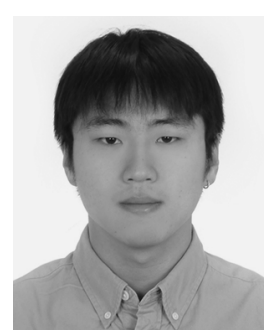

Seonghyun Jeon (S'06) was born in Seoul, Korea, in 1984 . He received the B.S. degree in electrical engineering and computer science from the Korea Advanced Institute of Science and Technology (KAIST), Daejeon, Korea, in 2006, and is currently working toward the M.S. degree at KAIST.

His research interest is body channel communication.

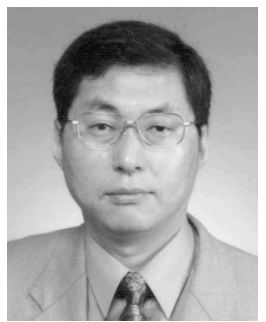

Hoi-Jun Yoo (M'95-SM'04) received the B.S. degree in electronics from Seoul National University, Seoul, Korea, in 1983, and the M.S. and Ph.D. degrees in electrical engineering from the Korea Advanced Institute of Science and Technology (KAIST), Daejeon, Korea, in 1985 and 1988, respectively. His doctoral research concerned the fabrication process for $\mathrm{GaAs}$ vertical opto-electronic integrated circuits.

From 1988 to 1990, he was with Bell Communications Research, Red Bank, NJ, where he invented the 2-D phase-locked vertical-cavity surface-emitting laser (VCSEL) array, the front-surface-emitting laser, and the high-speed lateral HBT. In 1991, he became Manager of the DRAM Design Group, Hyundai Electronics, and designed a family of fast-1 MDRAMs and synchronous DRAMs, including a 256M SDRAM. From 1995 to 1997, he was a faculty member with Kangwon National University. In 1998, he joined the faculty of the Department of Electrical Engineering, KAIST. In 2001, he founded the System Integration and IP Authoring Research Center (SIPAC), a national research center, funded by the Korean Government to promote worldwide IP authoring and its system-on-chip (SoC) application. From 2003 to 2005, he was the Project Manager for SoC with the Korea Ministry of Information and Communication. He authored DRAM Design (Hongleung, 1996; in Korean) and High Performance DRAM (Sigma, 1999; in Korean). His current interests are SoC design, IP authoring, high-speed and low-power memory circuits and architectures, design of embedded memory logic, opto-electronic integrated circuits, and novel devices and circuits.

Dr. Yoo was the recipient of the 1994 Electronic Industrial Association of Korea Award for his contribution to DRAM technology and the 2002 Korea Semiconductor Industry Association Award. 